\title{
Two-mode chaos and its synchronization properties
}

\author{
Postnov, D.E.; Shishkin, A.V.; Sosnovtseva, Olga; Mosekilde, Erik
}

Published in:

Physical Review E

Link to article, DOI:

10.1103/PhysRevE.72.056208

Publication date:

2005

Document Version

Publisher's PDF, also known as Version of record

Link back to DTU Orbit

Citation (APA):

Postnov, D. E., Shishkin, A. V., Sosnovtseva, O., \& Mosekilde, E. (2005). Two-mode chaos and its synchronization properties. Physical Review E, 72(5), 056208. https://doi.org/10.1103/PhysRevE.72.056208

\section{General rights}

Copyright and moral rights for the publications made accessible in the public portal are retained by the authors and/or other copyright owners and it is a condition of accessing publications that users recognise and abide by the legal requirements associated with these rights.

- Users may download and print one copy of any publication from the public portal for the purpose of private study or research.

- You may not further distribute the material or use it for any profit-making activity or commercial gain

- You may freely distribute the URL identifying the publication in the public portal

If you believe that this document breaches copyright please contact us providing details, and we will remove access to the work immediately and investigate your claim 


\title{
Two-mode chaos and its synchronization properties
}

\author{
D. E. Postnov, ${ }^{1}$ A. V. Shishkin, ${ }^{1}$ O. V. Sosnovtseva,${ }^{2}$ and E. Mosekilde ${ }^{2}$ \\ ${ }^{1}$ Physics Department, Saratov State University, Astrakhanskaya Street 83, Saratov, 410026, Russia \\ ${ }^{2}$ Department of Physics, The Technical University of Denmark, 2800 Kongens Lyngby, Denmark
}

(Received 22 December 2004; revised manuscript received 7 April 2005; published 14 November 2005)

\begin{abstract}
Using a simple model with bimodal dynamics, we investigate the intra- and inter-system entrainment of the two different time scales involved in the chaotic oscillations. The transition between mode-locked and modeunlocked chaos is analyzed for a single system. For coupled oscillators, we demonstrate full and partial synchronization patterns depending on the adjustment between the fast and slow time scales and reveal the embedded structure of the corresponding synchronization regions.
\end{abstract}

DOI: 10.1103/PhysRevE.72.056208

PACS number(s): 05.45.Xt

Synchronization of chaotic systems has become a major field of research in recent years [1,2]. Different criteria for and approaches to chaotic synchronization have been developed and peculiarities of the bifurcation mechanisms involved in the desynchronization have been examined [3-10]. It appears that the classical concept of synchronization related to the locking of the basic frequencies and instantaneous phases of regular oscillations (and to the suppression of the basic frequencies at high forcing amplitudes) is also applicable to a certain class of chaotic systems, namely, to systems for which the basic frequency can easily be distinguished in the power spectrum [7,9]. This frequency is typically related to the period of the original limit cycle from which the chaotic attractor is produced via a cascade of period-doubling bifurcations (the Feigenbaum scenario).

While entrainment of single-mode deterministic or stochastic oscillations is relatively well-understood, the dynamics of systems with several oscillatory modes is less studied. Living systems often exhibit oscillations with different time scales. The thalamocortical relay neurons, for instance, can generate either spindle or delta oscillations [11]. Neiman and Russell [12] have recently found that the electroreceptors in paddlefish can be biperiodic. Elston et al. [13] have studied transitions between synchrony and asynchrony in both slow oscillations and fast spikes for a pair of biological neurons. Most recently, Sosnovtseva et al. [14] have described the individual nephron as a two-mode oscillator demonstrating relatively fast oscillations associated with the myogenic regulation of the arteriolar diameter and slower oscillations related to a delay in the tubuloglomerular feedback.

Many models of bursting neurons [15], for example, can be split into slow and fast subsystems. Such an approach works perfectly when the subsystems can operate separately and the coupling is weak. Otherwise, the paradigm of coupled units seems to be less fruitful. Hence the description of double-oscillatory nature of the original system by means of a single two-mode oscillator is useful when (i) coupling is strong enough and (ii) the essential dynamical effects arise due to interaction between the subsystems.

In this paper we show how the synchronization properties of coupled oscillators that individually demonstrate twomode chaotic behavior are related to intraoscillator transitions in the chaotic regime.

The model we consider consists of two oscillatory sub- units. A self-sustained oscillator drives a damped nonlinear oscillator via both additive and multiplicative forcing. From a physical point of view, this may be considered as a parametric excitation of the fast oscillations. Besides the abovementioned nephron system, from which our model was originally derived, the problem has clear implementations in terms, for instance, of nonlinear electronic circuits or coupled mechanical oscillators.

The set of equations reads:

$$
\begin{gathered}
\ddot{x}-\left(1-x^{2}\right) \dot{x}+\omega^{2} x=E+c \dot{v}, \\
\ddot{v}+d \dot{v}+v \Omega(v)=F(x, v),
\end{gathered}
$$

where the first equation represents a van der Pol-type oscillator with frequency $\omega$. This oscillator is subjected to a constant force $E$ and receives a feedback $c \dot{v}$ from the other subunit. The second equation describes a damped oscillator with a frequency $\Omega(v)$ represented by a nonlinear function of the form $\Omega(v)=1+\beta e^{v}$ with $\beta \ll 1$. This form originally derives from our nephron model but actually describes a fairly generic case: for small $v, \Omega(v) \approx 1$, but larger values of $v$ produce a considerable upshift of the resonance frequency. The term $F(x, v)$ represents the forcing from the first oscillator. The specific form to be used includes both an additive and a multiplicative forcing:

$$
F(x, v)=a \tanh (x)(1+\gamma v) .
$$

The hyperbolic tangent $\tanh (x)$ is used to represent saturation phenomena at both very positive and very negative values of $x$. Together with the nonlinear frequency term $\Omega(v), F(x, v)$ provides stabilization of the oscillation amplitude in the parametrically forced oscillator (2).

Throughout our study $\omega^{2}$ and $E$ are used as control parameters while the other parameters are fixed at $c=2.0$, $d=0.1, \beta=0.001, a=0.474$, and $\gamma=12.85$. The substitution $y=\dot{x}, u=\dot{v}$ is applied to convert the Eqs. (1) and (2) into a set of four first-order ODEs:

$$
\begin{gathered}
\dot{x}=y, \\
\dot{y}=\left(1-x^{2}\right) y-\omega^{2} x+E+c u, \\
\dot{v}=u,
\end{gathered}
$$




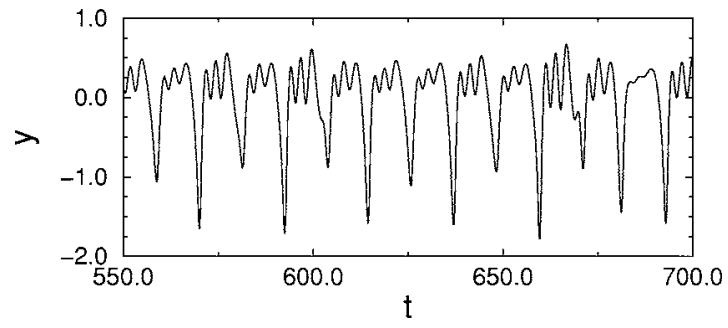

FIG. 1. Typical time series of two-mode chaos. $E=-0.4898$ and $\omega^{2}=0.5202$.

$$
\dot{u}=-d u-v \Omega(v)+F(x, v) .
$$

At vanishingly low values of $c$, the self-sustained dynamics of the system is bounded by the lines of an Andronov-Hopf bifurcation for subsystem (4) and (5):

$$
E= \pm \omega^{2} \text {. }
$$

However, for finite values of $c$, self-sustained regimes occupy a wider region on the $\left(\omega^{2}, E\right)$ parameter plane because of the positive feedback provided by the term $c u$. For larger $c$ values ( $c=2.0$ in our study), this region is closely covered by enumerous regular, quasiperiodic, and chaotic regimes. Among these regimes, we focus on the regime of chaotic dynamics (Fig. 1) that appears through a period-doubling cascade, but whose main feature is the presence of two time scales originating from the slow dynamics of subunit (4) and (5) and the fast dynamics of subunit (6) and (7). The phase and frequency for the second subunit can be estimated directly from the phase projection on the $(v, u)$ plane. For the first subunit (4) and (5), the corresponding phase projection contains a considerable contribution from the second subunit. To filter out the contribution from the fast component we introduce two auxiliary equations:

$$
\dot{\xi}=\omega(x-\xi) \text { and } \dot{\eta}=\omega(\xi-\eta) \text {. }
$$

In this way, we can extract information about each of the two oscillatory modes by calculating return times $\tau_{x}$ and $\tau_{v}$ of the phase trajectories to Poincaré secants defined by $\eta=0$ and $u=0$, respectively, in the $(\xi, \eta)$ and $(v, u)$ phase subspaces as discussed in Ref. [1]. Introducing the rotation number

$$
r=\left\langle\tau_{v}\right\rangle /\left\langle\tau_{x}\right\rangle
$$

we can determine the ratio between the slow and fast frequencies associated with the first and the second subunits.

Depending on the control parameters, $r$ takes rational or irrational values. Figure 2 presents a plot of $r$ vs $E$ for $\omega^{2}=0.5202$. The mode-locked regimes obviously correspond to the left branch of the curve $r=1 / 4$. Let us focus on the development of the two-mode chaotic attractor. With increasing $E$, chaos occurs at $E \approx-0.48989$, but the two modes remain locked and the individual system exhibits a perioddoubling chaos. At $E \approx-0.4898$, one observes a significant rise of the $r$ curve with further increase of $E$. Inspection of the system dynamics shows that there is a clear difference between chaotic attractors with rational and with floating rotation numbers. Evolution of phase diffusion coefficient $d$ [16] along $E$ reveals the same transition. We will refer to

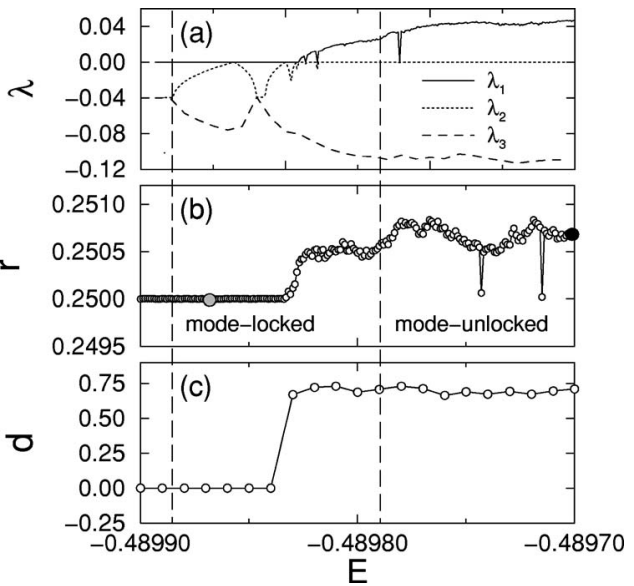

FIG. 2. (a) The three largest Lyapunov exponents, (b) rotation number $r$, and (c) phase diffusion coefficient $d$ as a function of control parameter $E$ for a single system with $\omega^{2}=0.5202$. While the largest Lyapunov exponent grows monotonically, the rotation number and phase diffusion coefficient indicate transitions inside the chaotic dynamics. The gray point represents an example of modelocked chaos. The black point corresponds to mode-unlocked chaos. The properties of the two types of chaos are compared in Fig. 3.

these attractors as "mode-locked" and "mode-unlocked" chaos, respectively. At the same time, no qualitative changes are found in the Lyapunov exponents.

In Fig. 3 the two columns of panels allow us to compare the attractor characteristics before and after the mode-unlocking transition at the points marked gray $(E=-0.48987)$ and black $(E=-0.48970)$ in Fig. 2. It is clearly seen that the $3 \mathrm{D}$ phase projection (top row) changes in a specific way by adding loops inside and around the main body of the attractor. The next row is a zoom on part of the attractor in the $(x, y)$ phase projection. The main difference between the two panels is the appearance in the right-hand panel of small-sized additional structures in the bundle of trajectories (indicating the existence of a time scale faster than the one that defines the main shape of the attractor). The third row shows a return time map with $\tau_{v}(n)$ being $\tau_{v}$ calculated $n$ times. For simple period-doubling chaos (left panel) this map has a clearly visible segmented structure (and there is an order of the segments to be visited). After the mode-unlocking transition (right panel), however, the map becomes more disordered with merging segments and with many points outside the main part of the map. With this, the power spectra reveal the band structure of the chaotic attractor. Note that the transition we discuss occurs for perioddoubling chaos, thus the well-known band-merging bifurcations would be expected to occur. However, in our case, the sequence of band-merging bifurcations is interrupted by the above-described mode-unlocking transition. The two bottom rows indicate the changes in the distribution of return times $\tau_{x}$ and $\tau_{v}$. Before the mode-unlocking transition there is a well-pronounced band structure for both time scales. After the transition, the histogram for the slow time scale becomes uniform but clearly bounded. For the fast time scale, the histogram remains split in a few segments and spread over a wider interval. Summarizing the above description, the tran- 

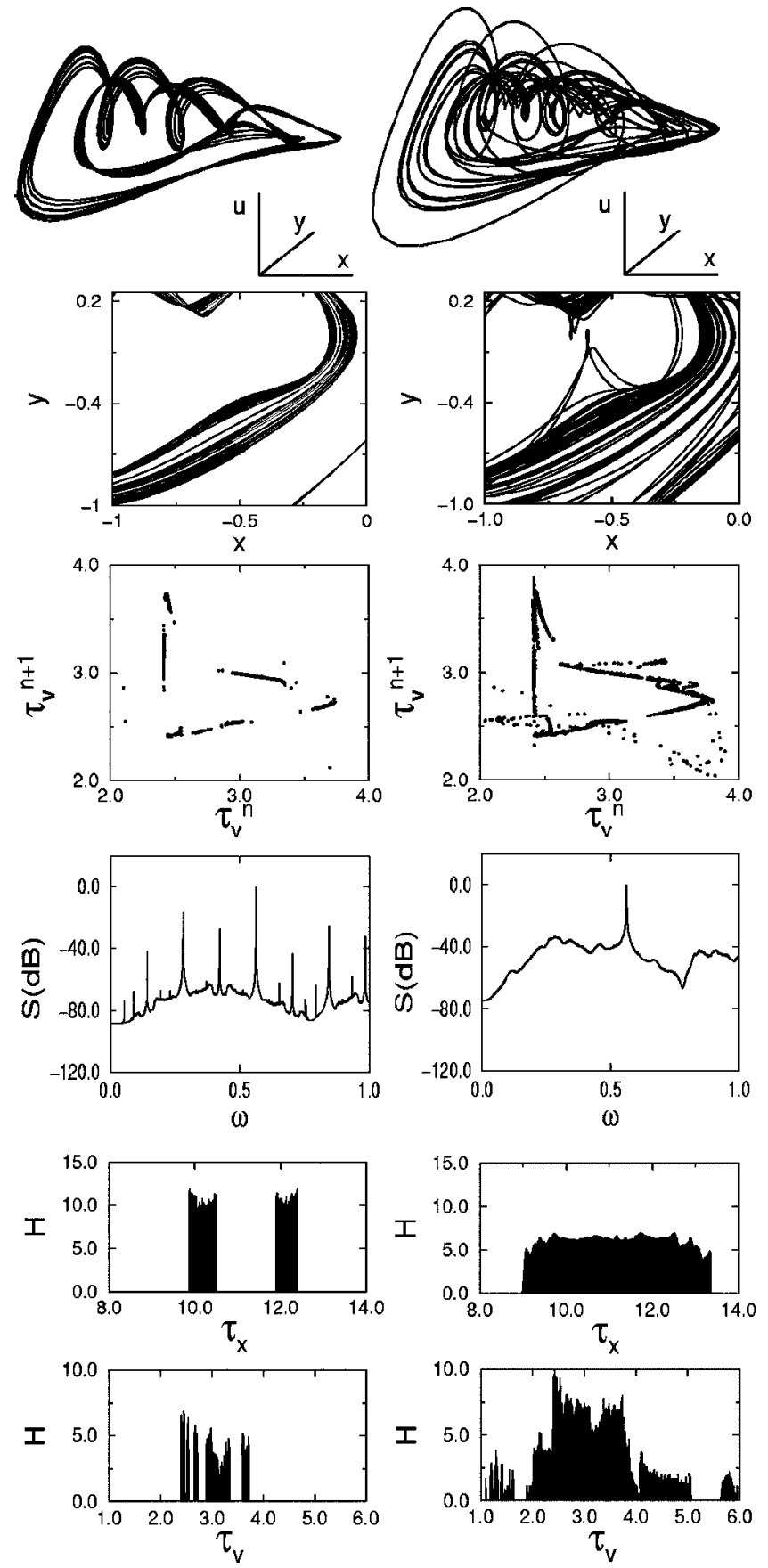

FIG. 3. Comparison of chaos characteristics at $E=-0.48987$ (left panels) and at $E=-0.48970$ (right panels) corresponding to the gray and black points in Fig. 2, respectively. There are obvious differences in the two-mode chaos before and after the modeunlocking transition.

sition from a resonant value of $r$ to floating behavior is accompanied by considerable changes in attractor characteristics as indicated in Fig. 3.

A similar phenomenon was observed in a case of generalized synchronization of two chaotic systems with frequency ratio 1:2 [18]. However, in our case the system cannot be split into chaotic oscillators and chaos appears to nonlinear interaction between functional units.

We have also compared the observed transition with the known evolution of a chaotic attractor to so-called "Shilnikov chaos" [17]. Again a clear difference exists. In our case the trajectory does not visit the close vicinity of an unstable equilibrium point embedded in the attractor. At least, there are no visible changes before and after mode-unlocking transition. Accordingly, the statistics of mean return times (given in the bottom rows of Fig. 3) is rather different from what we know for the Shilnikov attractor where the return time histogram extends to (infinitely) large times. In our case the return time histogram is smoothed but bounded for both modes.

Let us now consider how such systems, individually operating in the two-mode chaotic regime, can interact. We introduce a simple one-variable-difference coupling term with a strength $k$. The equation for the $x$ variable in Eq. (4) then becomes

$$
\dot{x}_{1}=y_{1}+k\left(x_{2}-x_{1}\right), \quad \dot{x}_{2}=y_{2}+k\left(x_{1}-x_{2}\right),
$$

where subscripts indicate the first and second interacting units. By calculating two rotation numbers each being the ratio between similar time scales in the coupled units,

$$
r_{x}=\frac{\left\langle\tau_{x 1}\right\rangle}{\left\langle\tau_{x 2}\right\rangle}, \quad r_{v}=\frac{\left\langle\tau_{v 1}\right\rangle}{\left\langle\tau_{v 2}\right\rangle},
$$

we can separately describe the adjustment of the slow and fast modes. The simplest way to introduce a mismatch between the units would be to choose different values of $\omega_{1}^{2}$ and $\omega_{2}^{2}$. However, for the individual system (4)-(7) the curves of period-doubling bifurcation are generally parallel to the Hopf bifurcation curve given by Eq. (8), and any variation of $\omega^{2}$ will change not only the main frequency but also the operating regime of the unit. Hence it would be difficult to come to a reasonable conclusion about the interaction between the attractors of a particular type. To avoid this problem, as suggested in Ref. [7], we have introduced a mismatch through an additional scale factor $\varepsilon$ in the lefthand side of the equations for one of the interacting units: $\varepsilon=1.0$ corresponds to the case of identical units, while variations below and above 1.0 give rise to a detuning that does not influence the operating regime.

Synchronization of one-mode chaotic systems via frequency/phase locking is thoroughly studied [1,2,9]. However, entraiment of two-mode chaotic regimes occurs in a more complex way. Let us consider the mutual adjustment of the oscillatory modes for the selected value of the coupling strength $k=0.0035$. Figure 4 presents the variation of the rotation numbers for the slow $r_{x}$ and fast $r_{v}$ time scales versus the frequency mismatch $\varepsilon$. There exists an interval of $\varepsilon \in[0.9984,1.00176]$ where $r_{x}=r_{v}=1.0$. This implies synchronous behavior with respect to both time scales. Both for larger and for smaller values of $\varepsilon$, the rotation number $r_{v}$ diverges from 1.0 while $r_{x}$ remains equal to one within a wider interval of $\varepsilon \in[0.9957,1.003$ 82]. This demonstrates desynchronization between the fast oscillatory modes in the coupled units while the slow modes remain locked. In this way, both partial synchronization (one of two time scales is synchronized) and all-mode frequency locking of chaos can be observed. Note that the behaviors of $r_{x}$ and $r_{v}$ near the edges of the locked region are different. Comparing the 


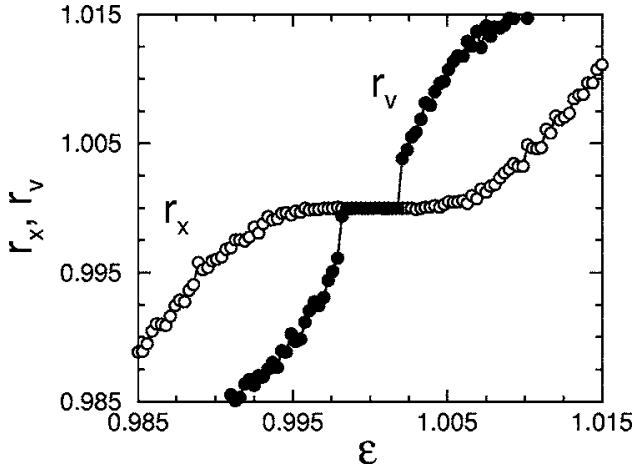

FIG. 4. Adjustment of two pair of oscillatory modes is indicated by changes in the $r_{x}$ and $r_{v}$ rotation numbers with respect to the frequency mismatch $\varepsilon$. $\omega_{2}^{2}=0.5202, \quad E_{1}=E_{2}=-0.48987$, and $k=0.0035$.

shape of the curves with the known synchronization pictures, one can draw analogies with the synchronization of periodic oscillations (sharp tongue edges imply saddle-node bifurcations) for $r_{v}$ and something similar to chaotic phase synchronization or synchronization of noisy oscillations for $r_{x}$ [1]. We assume that this reflects different synchronization mechanisms for the fast and slow modes.

Figure 5 shows the synchronization regions on the $(\varepsilon, k)$ parameter plane for the slow and fast oscillatory modes separately. We now clearly see that the two time scales have different widths of the Arnold tongues down to vanishingly small coupling strengths. An interesting observation can also be made for stronger coupling. For $k>0.004$, the fast oscillatory mode is completely desynchronized and displays a gradual increase of $r_{v}$ with increasing $\varepsilon$. This seems to be due to coupling through the slow $x$ variable. A stronger coupling increases the coupling-induced shift of the operating point for the two interacting units and, hence, provokes the complete unlocking of the fast modes from the slow ones. Since the fast modes can interact only via the slow variable such a situation leads to desynchronization.

In conclusion, we have reported a new mode-unlocking transition for chaos that has a significant influence on the cooperative dynamics of the coupled units. Separate-mode synchronization in the form of full and partial entrainments of the involved time scales can be diagnosed. Although obviously connected, the mutual behaviors of the slow and fast
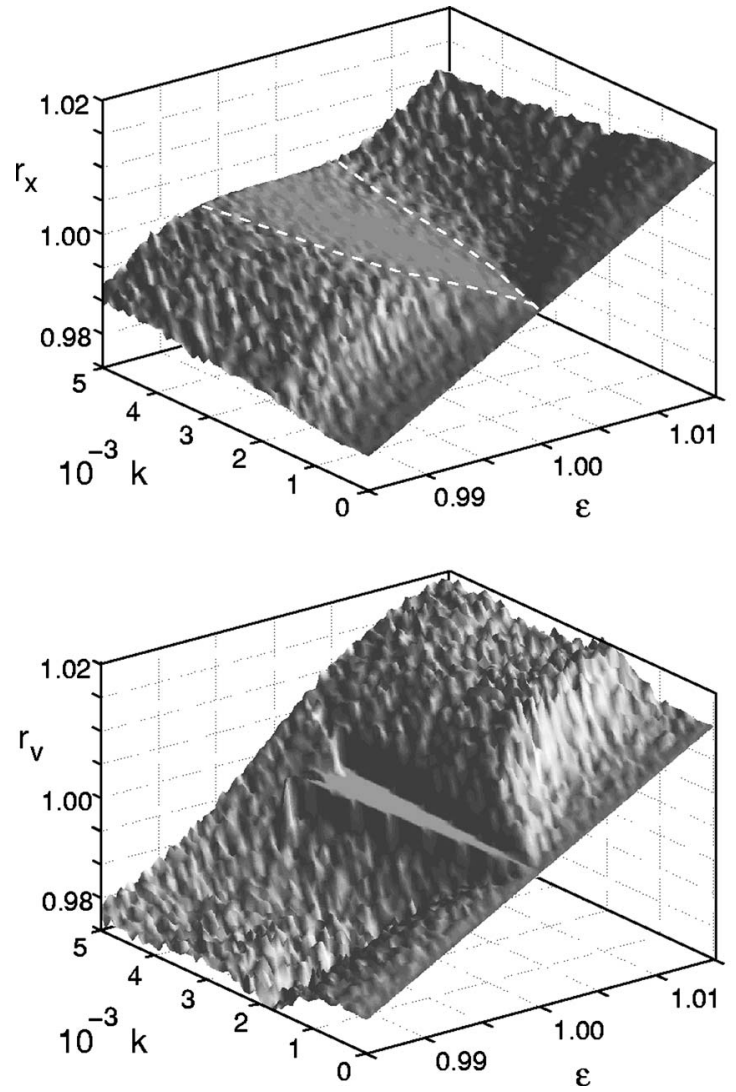

FIG. 5. 3D plots on the (frequency mismatch $\varepsilon$ vs coupling strength $k$ ) parameter plane for rotation numbers $r_{x}$ and $r_{v}$ separately. $\omega_{1}^{2}=\omega_{2}^{2}=0.5202$ and $E_{1}=E_{2}=-0.48987$.

modes manifest many signs typical for independent time scales, including resonances, quasiperiodicity, and chaos. Our results contribute to the understanding of how the recently discovered mechanisms of chaotic phase synchronization operates in more complex and high-dimensional systems.

This work is part of research activities of the EU supported NoE in Biosimulation (Contract No. 005137). D.P. acknowledges support from RFBR Grant 04-02-16769 and the Otto Monsteds Fond.
[1] A. Pikovsky, M. Rosenblum, and J. Kurths, Synchronization: A Universal Concept in Nonlinear Sciences (Cambridge University Press, Cambridge, England, 2001).

[2] E. Mosekilde, Y. Maistrenko, and D. Postnov, Chaotic Synchronization: Applications to Living Systems (World Scientific, Singapore, 2002).

[3] H. Fujisaka and Y. Yamada, Prog. Theor. Phys. 69, 32 (1983).

[4] V. S. Afraimovich, N. N. Verichev, and M. I. Rabinovich, Radiophys. Quantum Electron. 29, 795 (1986).

[5] L. M. Pecora and T. L. Carroll, Phys. Rev. Lett. 64, 821
(1990)

[6] G. Dykman, P. Landa, and Y. Neimark, Chaos, Solitons Fractals 1, 339 (1992).

[7] V. S. Anishchenko, T. E. Vadivasova, D. E. Postnov, and M. A. Safonova, Int. J. Bifurcation Chaos Appl. Sci. Eng. 2, 633 (1992).

[8] N. F. Rulkov, M. M. Sushchik, L. S. Tsimring, and H. D. I. Abarbanel, Phys. Rev. E 51, 980 (1995).

[9] M. G. Rosenblum, A. S. Pikovsky, and J. Kurths, Phys. Rev. Lett. 76, 1804 (1996). 
[10] V. S. Anishchenko, A. N. Silchenko, and I. A. Khovanov, Phys. Rev. E 57, 316 (1998).

[11] X.-J. Wang, Neuroscience (Oxford) 59, 21 (1994).

[12] A. Neiman and D. F. Russell, Phys. Rev. Lett. 86, 3443 (2001).

[13] R. C. Elson, A. I. Selverston, R. Huerta, N. F. Rulkov, M. I. Rabinovich, and H. D. I. Abarbanel, Phys. Rev. Lett. 81, 5692 (1998).

[14] O. V. Sosnovtseva, A. N. Pavlov, E. Mosekilde, and N.-H.
Holstein-Rathlou, Phys. Rev. E 66, 061909 (2002).

[15] J. Keener and J. Sneyd, Mathematical Physiology (Springer, New York, 1998).

[16] A. Neiman, A. Silchenko, V. Anishchenko, and L. Schimansky-Geier, Phys. Rev. E 58, 7118 (1998).

[17] V. S. Anishchenko, Dynamical Chaos-Models and Experiments (World Scientific, Singapore, 1995).

[18] N. F. Rulkov and C. T. Lewis, Phys. Rev. E 63, 065204(R) (2001). 